\title{
Gene therapy: New Horizon in Orthopaedic Management
}

\author{
Provash Chandra Saha, ${ }^{1}$ Sushmita Roy, ${ }^{2}$ KrishnaPriya Das ${ }^{3}$ \\ ${ }^{I}$ National Institute of Traumatology and Orthopaedic Rehabilitation, Dhaka, Bangladesh, \\ ${ }^{2}$ Department of Microbiology, Enam Medical College, Savar, Dhaka, Bangladesh \\ ${ }^{3}$ Department of Orthopaedic surgery, Bangabondhu Sheikh Mujib Medical University, Dhaka, Bangladesh.
}

\begin{abstract}
Gene transfer technologies enable the controlled, targeted and sustained expression of gene products at precise anatomical locations, such as the joint. In this way, they offer the potential for moreeffective, lessexpensive treatments of joint diseases with fewer extra-articular adverse effects.Many orthopaedic conditions are difficult to treat by conventional means; however, they are good candidates for gene therapy.Arthritis, aseptic loosening of prosthetic joints, bone defects, orthopaedic tumours, as well as the repair and regeneration of cartilage, ligaments, and tendons-in all these conditions gene therapy through transgene expressionmay have attractive implications. There have been few clinical trials and no FDA-approved gene therapy product exists.Among the multifactorial reasons, clinical translation is expensive anddifficult to fund by traditional academic routes, gene therapy is viewed as unsafe andrisky. Nevertheless, the preclinical and early clinical data are impressive and provide considerable optimism that gene therapy will provide straightforward, effective solutions to the clinical management of several common debilitating orthopaedic disorders that are otherwise difficult and expensive to treat.
\end{abstract}

Keywords: Gene therapy, Gene transfer, Orthopaedic conditions, Transgene expression. Correspondence:Dr.Provash Chandra Saha. Medical Officer, NITOR, Bangladesh,

\section{Introduction}

Gene Therapynow-a-days still exist experimental, attempt to modify genetic structure or gene encoding.Ittargets germ line gamete or somatic cells, using vectors to deliver genes within cells. Germ line gene therapy aims to prevent a genetic disorder from passing to new generations, while somatic gene therapy targets genetic disorders that already exist in individuals. (Beltina.Org, Genetics and Molecular medicine). Treatment of diseases is designed by inserting genesinto an individual's cells and tissues, and in case of hereditary diseases a defective mutant allele is replaced with a functional one. In recent years, it has been shown that gene therapy is a promising technology to treat or even cure several chronic or even fatal diseases for which there is no attractive alternative therapy. ${ }^{1}$

Many orthopaedic conditions are highly prevalent, chronic, incurable, degenerative, and disabling; they impose considerable misery and economic burdens on vast population and severely reduce quality of life. An estimated 40 million Americans have arthritis, 28 million have osteoporosis, and >18 million suffer low-back pain. The cost of these conditions from medical care, lost working days, and related issues is astronomical$\$ 849$ billion/annum according to recent estimates. ${ }^{2}$

After 20 years of promises, still there is no FDA-approved gene product for orthopaedic management rather a few clinical trials, only one of which, for rheumatoid arthritis (RA), completed Phase II. ${ }^{3}$ The science behind orthopaedic gene therapy is generally sound, and the pre-clinical data are impressive, but unfortunately its clinical realization is very slow, still many orthopaedic conditions are well suited for gene therapies. ${ }^{4}$

\section{Vector System and Promoter- important machineries for gene therapy}

An important feature for any successful gene therapy protocol is the vector system. Both viral and nonviral vector based therapy systems are in use. Each of these vectors possesses a number of unique features, advantages and disadvantages. The most are classical adenoviral vectors. ${ }^{5}$ Retroviral vectors have also been used for transducingadult MesenchymalStem Cells (MSC) and osteoprogenitors but with relatively poor results. ${ }^{6}$ BMPs (bone morphogenetic Proteins) can alter the differentiation pathwaysof muscle progenitors as well asfat tissue stem cells towards the osteogenic pathways. ${ }^{7}$ Osteogenic differentiation of adipose-derived stem cells was induced by transfection with rhBMP2 ${ }^{8}$ Penget al ${ }^{9}$ for enhancing the secretion of BMP-4 transgene from transduced bone marrow adult MSCs ex vivo used an MFG-based, VSV-G(vesicular stomatitisvirus) pseudo typed retroviral vector.

Achieving high expression is not the only goal of stem cell- based gene therapy.Limited expression of the transgene is important in orthopaedic medicine for multi course therapy and is achieved by the use of tissuespecific promoters. Osteoblast- specific promoters will ensure expression in the bone marrow zone where active synthesis of bone matrix occurs likethe collagen 1 A1 promoter sequence in order to achieve osteoblasticspecific 
expression. Abboud et al used similar approach withosteocalcin promoterwhere osteoblasts were engineered to express CSF-1 for the treatment of osteopetrosis. For skeletal defects due to injury, trauma or time-limited diseases this approach is less relevant, as it maintains long-term expression in osteoblasts. ${ }^{10}$

Transgene expression and temporal control on the duration of expression may be critical in the future development of gene therapy applications for orthopaedic medicine. To overcome this, tetracycline-regulated promoters have been used to manage transgene expression in a model of MSC line, C3H10T1/2, expressing rhBMP-2. ${ }^{11}$ Such promoters have the potential to allow us to control the time and level of transgene expression and can be used osteoporosis, that may need long-term gene expression and regulation, and for local injury and time-limited disease.

\section{Gene Therapy in Orthopaedic Conditions}

In orthopaedic field, gene therapy might be clinically useful in Mendelian disorders; tumours; arthritis and other joint diseases; tissue repair and regenerative medicine (TERM).

\subsection{Arthritis and Other Joint Diseases}

Arthritis may well be due to an interaction between genetic and environmental factors, such that individuals with certain predisposing genetic factors are more likely to develop osteoarthritis when exposed to abnormal mechanical loading. ${ }^{12}$ Osteoarthritis is commonest, local, incurable, degenerative condition of a limited number of weight-bearing joints, especially the hips and knees. In contrast, rheumatoid arthritis (RA), the second most common form, is a systemic, autoimmune condition affecting multiple joints.

The local, intra-articular gene therapy has entered clinical trials. The intra- and peri-articular tissues have the potential to synthesize anti-arthritic gene products, thereby providing a sustained, local therapy for individual arthritic joints. This approach is attractive because joints are discrete, accessible cavities that can be readily injected. Genetically modified synovial cells then express the transgene and deliver secreted transgene products into the joint. Therapeutic complementary DNA (cDNA) is incorporated into a vector, which is used to deliver the transgene by direct intra-articular injection (in vivo delivery) or by modifying cells that are subsequently introduced into the joint (ex vivo delivery). ${ }^{13}$

There is an ability of certain cells, particularly dendritic cells, macrophages, and lymphocytes, to home to sites of inflammation. Cells of this type can thus deliver a genetic payload selectively to inflamed joints following a single, parenteral application. This approach is known as facilitated local therapy. ${ }^{14}$

The first human application in arthritis gene therapy used an ex vivo protocol involving a retrovirus (MFGIRAP) to deliver the human interleukin-1 antagonist (IL-1Ra) cDNA to the metacarpophalangeal joints (knuckles) of subjects with RA. ${ }^{15}$ This study confirmed the feasibility and, in a preliminary fashion, safety of gene transfer to human, arthritic joints, and demonstrated the intra-articular expression of a biologically active transgene product. ${ }^{16}$

\subsection{Bone Cancer}

Most osteosarcomas involve mutations in p53 and the retinoblastoma gene (Rb), so there is obvious interest in using cDNAs encoding p53 and Rbfamily members, as well as conditionally replicating adenovirus. ${ }^{17}$ Recombinant adenovirus is frequently used for cancer gene therapy; Gu et al. ${ }^{18}$ interestinglynoted increased expression of the coxsackie virus and adenovirus receptor in several orthopaedic tumours, including osteosarcoma and Ewing's sarcoma. Coxsackie virus and adenovirus receptor expression correlated with the response of human osteosarcoma cells to Ad.p53 (ref. ${ }^{19}$ ). Osteosarcoma, the most common bone cancer that has been the subject of preliminary studies (reviewed in ref. ${ }^{20}$ ) using cDNAs encoding IL-12, IL-18, and IL-13 (ref. $\left.{ }^{21}\right)$; herpes simplex virus-thymidine kinase- ganciclovir; ${ }^{22}$ and cytosine deaminase. ${ }^{23}$

\subsection{Osteoporosis}

Osteoporosis involves a pathological loss of mineral from the skeleton. This reflects the uncoupling of osteoblastic and osteoclastic processes. The systemic delivery of cDNAs encoding the secreted molecules IL$1 \mathrm{Ra}$ and osteoprotegerin, ${ }^{24}$ which block proliferation of osteoclast, has shown efficacy in rodent models of osteoporosis. A monoclonal antibody to the osteoclast differentiation factor RANK ligand and several additional biologics are also in trial.

\subsection{Joint Replacement Surgery}

In orthopaedic surgery, artificial joint replacement is a common and successful arena of surgical modality. Although it is noted, about $10 \%$ of artificial hips and knees fail within 10 years as a result of aseptic loosening. ${ }^{25}$ 'Aseptic loosening' may occur due to the generation of wear debris which activates phagocytes. These cells release osteoclastic factors that induce a characteristic zone of osteolysis around the 
implant. Gene transfer can address this problem by delivering cDNAs whose products interfere with the response of the phagocyte or which antagonize the mediators produced by activated cells. ${ }^{26}$

Huizinga's group has developed an interesting gene based strategy for treating aseptic loosening that does not involve major surgery or replacing the prosthesis. ${ }^{14}$ Huizinga's approach uses a first-generation adenovirus to deliver nitroreductasecDNA to cells within the pseudosynovium (surrounded the loosened joint), which is then ablated by administration of the prodrug CB1954. ${ }^{27}$

\subsection{Degenerative Disease}

Intervertebral Disc Degeneration is highly prevalent degenerative disease with age for which surgical palliation is sometimes inadequate. Several clinical approaches are observed, using recombinant bone morphogenetic protein-7 (BMP-7; OP-1), recombinant growth and differentiation factor-5 [GDF-5, BMP-14, and Cartilage derived morphogenetic protein-1 (CDMP-1)]. Gene transfer is an attractive alternative to the repeated intradiscal injection of recombinant proteins. ${ }^{28}$ The cells within the disc do not normally divide. The disc is a dense, nonvascularized, alymphatic tissue and immunologically isolated and can support extended periods of transgene expression.

\subsection{Defective Bone Healing}

Bone has capability to heal naturally as well as bone healing is a greater problem resulting non-union. Stem cells have the self-renewal ability, and the abilityto give rise to differentiating cells. Adult MSCs and osteoprogenitors are relativelyeasy to isolate from the bone marrow and culture in vitroand to use them as vehicles for the deliveryof therapeutic genes in vivo, a strategy known as cellmediatedgene therapy. ${ }^{29}$

Primaryadult MSCs and C3H10T1/2 and primary marrow derived stem cells are employed for the delivery of growth factors of the BMP family especially bone morphogeneticprotein-2 (BMP-2), a highly osteoinductive agent for promoting osteogenic differentiation and bone formation. The segmental defect of a rat model was healed as effectively as those treated with recombinant, human BMP-2. ${ }^{30}$

Utilizing adult MSCs as vehicles for gene delivery hasan additional benefit over direct in vivo delivery ofproteins or genes. Engineered adult MSCs can potentiallyengraft into the damaged tissue in vivo and expressthe therapeutic genes for long periods, whereas local, onetime administration of genes or protein will have a limited time effect. ${ }^{31}$

Yet another benefit is that in thehealing process following transplantationof MSCs engineered to express rhBMP-2, and produce bone in an organized manner by augmenting new cartilage andbone on top of the defect edges, forming continuousregeneration between the original defect edges and thenewly formed bone. ${ }^{32} \mathrm{In}$ comparison, rhBMP-2protein delivery or the implantation of non-MSCs (CHO cells) expressing rhBMP-2 resulted in the formationof diffuse osseous foci with no continuity to theoriginal bone. ${ }^{29}$

\subsection{Stem-cell-based gene therapy applications for tendon and ligament}

Several studies have explored the avenue of gene therapy for the repair of ligament and tendon.Myoblasts, a more specific muscle cell progenitor,were employed. When engineered myoblasts wereinjected into the anterior cruciate ligament they integratedinto the tissue and maintained transgene expression, but retained their muscle phenotype. ${ }^{33}$

\subsection{Stem-cell-based gene therapy applications for cartilage}

Cartilage, unlike bone, is a tissue known for its difficulty in self-repair.Possible reasons for deficient self-regeneration of cartilage tissue are poorvascularization, insufficient stem cell recruitment andpersistent chronic inflammation.Cell-mediated gene therapy strategies for these diseases can be roughly divided into two approaches. The delivery of the secreted IL-1 receptor antagonist gene was the most common strategy employed to counteract the inflammatory process.Insulin-like growth factor-1 (IGF-1), fibroblasts engineered transforming growthfactor-b1 (TGF-b1), BMP-2 in fibroblasts and chondrocytes had been used for the promotion of cartilage formation after injury. ${ }^{34}$

\section{Obstacles in clinical progress}

The field of gene therapy has a bad name. It is seen as risky, unsafe and, until recently, unable to deliver. The safety issue is particularly pertinent for orthopaedic applications, and the risk: benefit ratio is skewed. ${ }^{35}$ Despite progress in all of the areas, translating experimental gene therapy methods into regenerative surgery techniques for use in the operating room will remain a difficult challenge. The perception of risk makes industry, especially large pharmaceutical companies, reluctantto invest in orthopaedic gene therapy. This presents a major obstacle to progress, because itsclinical translation is extremely expensive. ${ }^{36} \mathrm{After}$ two decades, regenerative surgery is an adolescent looking forward to growing up," Dr.Giatsidis and co-authors write. 
"Despite extensive preclinical approaches, translation of gene therapy strategies into clinical trials is still a difficult and expensive process." ${ }^{37}$

\section{Conclusion}

Early-phase human clinical trials have been successfully conducted and others are in progress. Additional research is necessary to optimize gene transfer technologies and achieve regulated transgene expression. However, the most urgent need is for interventional studies in human disease and the funding with which to implement them. With sufficient resources and regulatorypragmatism, arthritis gene therapy stands a good chance of success.If this can be turned into evidence of success, even in a fewpatients, it is likely that the enthusiasm the orthopaedic community for novelty will supplythe needed momentum for further development.The high burden of orthopaedic disorders makes it imperative for getting the most promising gene therapeutic approaches from the research laboratory to the clinical practical field.

\section{Reference}

[1] E. Marshall. Gene therapy death prompts review of adenovirus vector. Science, 286, 1999, 2244-2245

[2] Anonymous Burden of musculoskeletal diseases in the United States. Bone and Joint Decade Newsletter, 1,2008,1.

[3] P.J. Mease, N. Wei, E.J. Fudman, A.J. Kivitz, J. Schechtman, R.G. Trapp, K.F. Hobbs, et al. Safety, tolerability, and clinical outcomes after intraarticular injection of a recombinant adeno-associated vector containing a tumor necrosis factor antagonist gene: results of a phase 1/2 Study. J Rheumatol.37, 2010, 692-703. [PubMed: 20032102]

[4] C.H. Evans. Gene therapy for bone healing.Expert Rev Mol Med.12, e18, 2010. [PubMed: 20569532]

[5] G. Turgeman. Engineered human mesenchymal stem cells: a novel platform for skeletal cell mediated gene therapy. J Gene Med, 3, 2001, 240-251.

[6] T. Engstrand. Transient production of bone morphogenetic protein 2 by allogeneic transplanted transduced cells induces bone formation. Hum Gene Ther, 11, 2000, 205-211.

[7] S. Ebara, K. Nakayama .Mechanism for the action of bone morphogenetic proteins and regulation of their activity.Spine, 27S, $2002,10-15$.

[8] J.L. Dragoo. Bone induction by BMP-2 transduced stem cells derived from human fat. J Orthop Res, 21, 2003, 622-629.

[9] H. Penget al. Development of an MFG-based retroviral vector system for secretion of high levels of functionally active humanBMP4. MolTher, 4, 2001, 95-104.

[10] S.L. Abboud. Rescue of the osteopetrotic defect in op/op mice by osteoblast-specific targeting of soluble colony stimulating factor. Endocrinology, 143, 2002, 1942-1949.

[11] I.K. Moutsatsos. Exogenously regulated stem cell mediated gene therapy for bone regeneration. MolTher, 3, $2001,449-461$.

[12] Osteoarthritis associated genes: an interview with Professor Jonathan Tobias, University of Bristol Published on July 2, 2013 at 5:25 AM, Interview conducted by April Cashin-Garbutt, BA Hons (Cantab)

[13] E. Gouze E. Transgene persistence and cell turnover in the diarthrodial joint: implications forgene therapy of chronic joint diseases. Mol. Ther., 15,2007, 1114-1120. [PubMed: 17440444]

[14] C.H. Evans, S.C Ghivizzani, P.D Robbins. Orthopedic Gene Therapy in 2008.The American Society of Gene Therapy. 265, 2008, $1-14$.

[15] C.H. Evans, P.D. Robbins, S.C. Ghivizzani, J.H. Herndon, R. Kang, A.B. Bahnson, et al. Clinical trial to assess the safety, feasibility, and efficacy of transferring a potentially anti-arthritic cytokine gene to human joints with rheumatoid arthritis.Hum Gene The, 7, 1996, 1261-1280.

[16] C.H. Evans, P.D. Robbins, S.C. Ghivizzani, M.C. Wasko, M.M. Tomaino, R. Kang, et al. Gene transfer to human joints: progress toward a gene therapy of arthritis. ProcNatlAcadSci USA, 102, 2005, 8698-8703.

[17] A.M. Witlox, V.W. Van Beusechem, B. Molenaar, H.Bras, G.R.Schaap, R. Alemany, et al. (2004). Conditionally replicative adenovirus with tropism expanded towards integrins inhibits osteosarcoma tumor growth in vitro and in vivo. Clin Cancer Res, 10, 2004, 61-67.

[18] W. Gu, A. Ogose, H. Kawashima, M. Ito, T Ito, A. Matsuba, et al. High- level expression of the coxsackievirus and adenovirus receptor messenger RNA in osteosarcoma, Ewing's sarcoma, and benign neurogenic tumors among musculoskeletal tumors. Clin Cancer Res, 10, 2004, 3831-3838.

[19] H. Kawashima, A.Ogose, T. Yoshizawa, R. Kuwano, Y. Hotta, T. Hotta, T et al. Expression of the coxsackievirus and adenovirus receptor in musculoskeletal tumors and mesenchymal tissues: efficacy of adenoviral gene therapy for osteosarcoma. Cancer Sci, 94 , $2003,70-75$.

[20] M.A. Witlox, M.L. Lamfers, P.I. Wuisman, D.T. Curiel G.P. Siegal. Evolving gene therapy approaches for osteosarcoma using viral vectors: review. Bone, 40, 2007, 797-812.

[21] C. Liebau, C. Roesel, S. Schmidt, C. Karreman, J.B. Prisack, JB, H. Bojar, et al. Immunotherapy by gene transfer with plasmids encoding IL-12/IL-18 is superior to IL-23/IL-18 gene transfer in a rat osteosarcoma model. Anticancer Res, 24, $2004,2861-2867$.

[22] Skotzko, M, Wu, L, Anderson, WF, Gordon, EM and Hall, FL (1995). Retroviral vector- mediated gene transfer of antisense cyclin G1 (CYCG1) inhibits proliferation of human osteogenic sarcoma cells. Cancer Res 55: 5493-5498.

[23] M. Ramnaraine, W. Pan, M. Goblirsch, C. Lynch, V. Lewis, P. Orchard, et al. Direct and by stander killing of sarcomas by novel cytosine deaminase fusion gene. Cancer Res 63, 2003, 68 47-6854.

[24] B. Bolon, C. Carter, M. Daris, S. Morony, C. Capparelli, A. Hsieh, et al. Adenoviral delivery of osteoprotegerin ameliorates bone resorption in a mouse ovariectomy model of osteoporosis. MolTher, 3, 2001, 197-205.

[25] J.N. Katz. Total joint replacement in osteoarthritis.BestPract Res ClinRheumatol, 20, 2006, 145-153.

[26] P.H. Wooley, E.M. Schwarz.Asepticloosening.Gene Ther, 11, 2004, 402-407.

[27] J.J. de Poorter, T.C. Tolboom, M.J. Rabelink, E. Pieterman, R.C. Hoeben, R.G. Nelissen, et al. Towards gene therapy in prosthesis loosening: efficient killing of interface cells by gene-directed enzyme prodrug therapy with nitroreductase and the prodrug CB1954. J Gene Med, 7, 2005, 1421-1428.

[28] C. Evans. Point of view.Spine33, 2008, 1517

[29] D. Gazit. Engineered pluripotent mesenchymal cells integrate and differentiate in regenerating bone: a novel cellmediated gene therapy. J Gene Med, 1, 1999, 121-133. 
[30] J.R. Lieberman. Regional gene therapy with a BMP-2- producing murine stromal cell line induces heterotopic and orthotopic bone formation in rodents. J Orthop Res, 16, 1998, 30-339.

[31] F. Gori . Differentiation of human marrow stromal cells: bone morphogenetic protein-2 increases OSF2/CBFA1, enhancesosteoblast commitment and inhibits late adipocyte maturation. J Bone Mineral Res, 14, 1999, 1522-1534.

[32] S.T. Yoon, S.D. Boden. Osteoinductive molecules in orthopaedics: basic science and preclinical studies. ClinOrthop, 395, 2002, 3343.

[33] J. Menetrey. Direct-, fibroblast- and myoblast-mediated gene transfer to the anterior cruciate ligament. Tissue Eng, 5, 1999,435442 .

[34] B.D. Brower-Tolandet al. Direct adenovirus-mediated insulin-likegrowth factor I gene transfer enhances transplant chondrocytefunction. Hum Gene Ther, 12,2001, 117-129.

[35] I.K. Moutsatsos. Exogenously regulated stem cell mediated gene therapy for bone regeneration. MolTher, 3, $2001,449-461$.

[36] C.H. Evans, S.C. Ghivizzani, P.D. Robbins. ORTHOPAEDIC GENE THERAPY - LOST IN TRANSLATION? Cell Physiol., 2012, 227(2), 416-420.

[37] Medical University of Vienna. Gene therapy as a new option for bone defects.ScienceDaily, 2012, December 7, Retrieved July 3, 2013, from http://www.sciencedaily.com 